\title{
The genome sequence of celery (Apium graveolens L.), an important leaf vegetable crop rich in apigenin in the Apiaceae family
}

Meng-Yao Li', Kai Feng ${ }^{1}$, Xi-Lin Hou', Qian Jiang ${ }^{1}$, Zhi-Sheng Xu' ${ }^{1}$, Guang-Long Wang ${ }^{1}$, Jie-Xia Liu', Feng Wang ${ }^{1}$ and Ai-Sheng Xiong ${ }^{1}$

\begin{abstract}
Celery (Apium graveolens L.) is a vegetable crop in the Apiaceae family that is widely cultivated and consumed because it contains necessary nutrients and multiple biologically active ingredients, such as apigenin and terpenoids. Here, we report the genome sequence of celery based on the use of HiSeq 2000 sequencing technology to obtain $600.8 \mathrm{~Gb}$ of data, achieving 189-fold genome coverage, from 68 sequencing libraries with different insert sizes ranging from $180 \mathrm{bp}$ to $10 \mathrm{~kb}$ in length. The assembled genome has a total sequence length of $2.21 \mathrm{~Gb}$ and consists of 34,277 predicted genes. Repetitive DNA sequences represent $68.88 \%$ of the genome sequences, and LTR retrotransposons are the main components of the repetitive sequences. Evolutionary analysis showed that a recent whole-genome duplication event may have occurred in celery, which could have contributed to its large genome size. The genome sequence of celery allowed us to identify agronomically important genes involved in disease resistance, flavonoid biosynthesis, terpenoid metabolism, and other important cellular processes. The comparative analysis of apigenin biosynthesis genes among species might explain the high apigenin content of celery. The whole-genome sequences of celery have been deposited at CeleryDB (http://apiaceae.njau.edu.cn/celerydb). The availability of the celery genome data advances our knowledge of the genetic evolution of celery and will contribute to further biological research and breeding in celery as well as other Apiaceae plants.
\end{abstract}

\section{Introduction}

Celery (Apium graveolens L.) is an annual or biennial herbaceous plant in the Apiaceae family that originated in the Mediterranean and the Middle East. It is a popular vegetable crop and is widely cultivated in Europe, East Asia, southeastern Oceania, and southern Africa (Fig. 1a). The whole celery plant exhibits aromatic flavor, and its leaf blades and petioles are the main edible organs (Fig. 1b). In addition to containing common nutrients such as vitamins, proteins and carbohydrates, celery

Correspondence: Ai-Sheng Xiong (xiongaisheng@njau.edu.cn)

${ }^{1}$ State Key Laboratory of Crop Genetics and Germplasm Enhancement, Ministry of Agriculture and Rural Affairs Key Laboratory of Biology and Germplasm Enhancement of Horticultural Crops in East China, College of Horticulture, Nanjing Agricultural University, 1 Weigang, Nanjing 210095, China

These authors contributed equally: Meng-Yao Li, Kai Feng contains flavonoids, carotenoids, terpenoids, and unsaturated fatty acids that exhibit biological activity and physiological functions in human beings ${ }^{1-4}$.

Flavonoids are a class of natural products that are widely found in plants, most of which exist in the form of glycosides. Vegetables and fruits are the predominant dietary sources of flavonoids. Flavonoids are one of the most important types of secondary metabolites in celery, mainly comprising apigenin, kaempferol, quercetin, and luteoli ${ }^{5}$. In particular, the content of apigenin in celery is higher than that in other plants ${ }^{6}$. Many studies on the isolation, identification, and application of flavonoids in celery have been carried out ${ }^{4,7,8}$. Apigenin and luteolin exhibit a wide range of pharmacological effects, including anti-bacterial, anti-oxidation, and cardiovascular protective effects ${ }^{9,10}$. Many terpenoids and aromatic compounds

\section{(c) The Author(s) 2020}

(c) (i) Open Access This article is licensed under a Creative Commons Attribution 4.0 International License, which permits use, sharing, adaptation, distribution and reproduction in any medium or format, as long as you give appropriate credit to the original author(s) and the source, provide a link to the Creative Commons license, and indicate if changes were made. The images or other third party material in this article are included in the article's Creative Commons license, unless indicated otherwise in a credit line to the material. If material is not included in the article's Creative Commons license and your intended use is not permitted by statutory regulation or exceeds the permitted use, you will need to obtain permission directly from the copyright holder. To view a copy of this license, visit http://creativecommons.org/licenses/by/4.0/. 


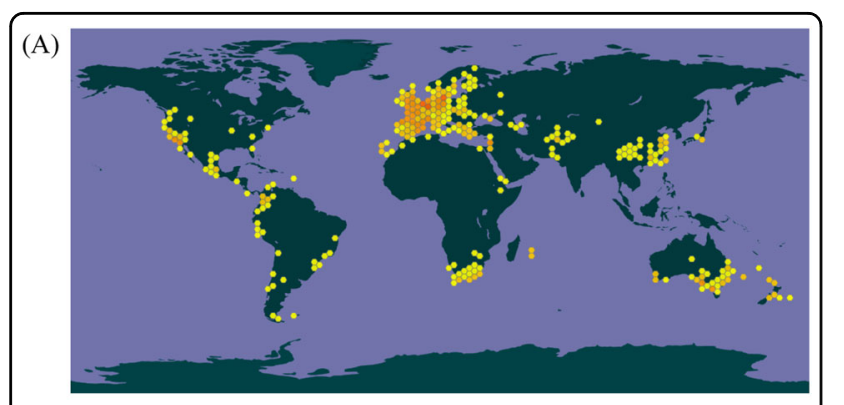

(B)

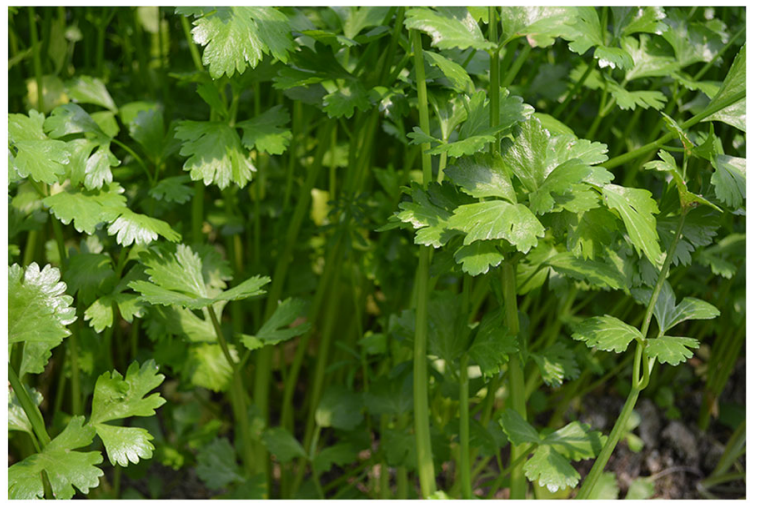

Fig. 1 Georeferenced records and an image of celery. a Georeferenced records of celery on the world map. $\mathbf{b}$ Image of celery. The data set was obtained from the GBIF website (www.gbif. org). The colored hexagons represent the locations of georeferenced celery records.

are present in celery tissues, which contribute to its unique fragrance. Some studies have shown that terpenoids from celery seed oil exert strong lethal effects on Aedes aegypti ${ }^{11}$. Effective celery components such as allergenic proteins, apigenin, and anthocyanin have caused wide public concern ${ }^{12-15}$. However, the regulatory mechanisms of the effective components of celery remain unclear.

Whole-genome sequencing has overcome the limitations of traditional basic research and generated massive data that greatly promote research on plants. The genome sequence of Arabidopsis thaliana was completed and published in 2000, providing the first reported sequencing data for higher plants ${ }^{16}$. Since then, the genomes of many species have been sequenced and published ${ }^{17-21}$. Apiaceae is one of the largest families of flowering plants, with 4000 species classified into 434 genera. The Apiaceae family contains several vegetable and spice crop species. However, the lack of complete genome data places greatly constrains the improvement of Apiaceae crops. The molecular resources for Apiaceae species are underdeveloped. Thus far, a reported carrot genome represents the only genome data for the Apiaceae family ${ }^{20,22}$.

In this paper, we report the genome sequence of celery, which is one of the most economically important species of the Apiaceae family. The $\sim 189 \times$ coverage sequence of the celery genome provides information on the overall organization, gene content, and structural components of the DNA. Transcription factors, disease resistance genes, apigenin and terpenoid biosynthesis-related genes, and other functional genes were also identified in this study. All of the data not only provide valuable resources for basic and applied research on celery but also lay the foundation for the analysis of the evolution and comparative genomics of celery and Apiaceae species.

\section{Materials and methods}

Plant material, DNA preparation, and genome sequencing

The celery material used in this study was the highly inbred line Q2-JN11, which was derived via the forced selfing of "Jinnan Shiqin". The seeds of Q2-JN11 were collected in the State Key Laboratory of Crop Genetics and Germplasm Enhancement, Nanjing Agricultural University, China. High-quality genomic DNA was extracted from the young leaves of celery for genome sequencing using a modified CTAB method ${ }^{23}$.

The genome was sequenced using the HiSeq 2000 platform. Libraries with six different insert sizes (180 bp, $500 \mathrm{bp}, 800 \mathrm{bp}, 2 \mathrm{~kb}, 5 \mathrm{~kb}$, and $10 \mathrm{~kb}$ ) were prepared from Q2-JN11 DNA and then sequenced according to standard Illumina protocols at the Beijing Genomics InstituteShenzhen (BGI-Shenzhen).

\section{Genome assembly}

The raw reads generated from each library were preprocessed to facilitate their assembly. Low-quality reads were filtered out, and reads with adapter contamination were removed using CutAdapt ${ }^{24}$. The paired-end reads were assembled into contigs using SOAPdenovo2 (http://soap. genomics.org.cn/soapdenovo.html ${ }^{25}$ with the multi-kmer option $(k$-mer $=63)$. We then aligned all usable pairedend reads onto the contig sequences and used mate-pair information on the order of the estimated insert size (180 bp to $10 \mathrm{~kb}$ ) to construct scaffolds. Gaps within the scaffolds were closed using GapCloser. BUSCO v3 was employed to measure the genome assembly using the Eudicotyledons (odb10) database with default parameters $^{26}$.

\section{Gene prediction and annotation}

The presence of possible celery-specific transcripts within a region was analyzed using Augustus 3.2.2 and $\mathrm{SNAP}^{27,28}$. For similarity-based gene prediction, five sequenced plants (A. thaliana, Mimulus guttatus, Solanum tuberosum, Solanum lycopersicum, and Daucus carota) were selected, and the protein sequences of these species were downloaded from Phytozome v12 (http:// www.phytozome.net). BLAST (identity $\geq 0.95$; coverage $\geq$ 0.90) was used to identify ESTs, mRNAs, and proteins with significant similarity to the celery genome sequence. 
The identity and coverage thresholds used in the alignment were determined according to the methods employed for the carrot and potato genomes ${ }^{20,29}$. Manual curation and several rounds of refinement using Exonerate v.2.2.0 were performed to realign or polish the sequences following filtering and clustering ${ }^{30}$. The functional annotation of protein-coding genes was achieved using BLASTP ( $E$ value of $1 \times 10^{-4}$ ) against the NCBI non-redundant protein sequence (nr), TrEMBL, SwissProt, Gene Ontology (GO), and Kyoto Encyclopedia of Genes and Genomes (KEGG) databases ${ }^{31,32}$. Blast2GO ${ }^{33}$ was used to obtain the relevant GO ID, and $\mathrm{WEGO}^{34}$ was applied to illustrate the distribution of gene classifications.

\section{Noncoding RNAs}

Noncoding RNAs were identified by searching against various RNA libraries. tRNA scan-SE (version 1.3.1) was employed to search for reliable tRNA positions ${ }^{35}$. Small nuclear RNAs (snRNAs) and microRNAs (miRNAs) were searched via a two-step method involving initial alignment with BLAST followed by searching with INFER$\mathrm{NAL}^{36}$ against the Rfam database (v12.0) ${ }^{37}$.

\section{Repetitive elements}

Transposable elements in the celery assembly were identified using a combination of homology-based and de novo approaches. The known repetitive elements were identified with RepeatMasker (version 3.3.0) against the Repbase library ${ }^{38}$. RepeatModeler (http://www. repeatmasker.org/RepeatModeler.html) was then used after masking the known repetitive elements. The consensus sequences generated with RepeatModeler were searched against the SWISS-PROT database using BLASTX, and consensus sequences with significant similarity to protein-coding genes were eliminated. Finally, RepeatMasker was run on the genome sequences using the consensus sequence as a library.

\section{Gene family analysis}

We used OrthoMCL to define a gene family as a group of genes descending from a single gene in the last common ancestor of the considered species ${ }^{39}$. The proteincoding genes of $M$. guttatus v2.0, S. lycopersicum iTAG2.4, D. carota v2.0, and A. thaliana TAIR10 were downloaded from Phytozome v12 (http://www. phytozome.net $)^{40}$. The longest protein sequence prediction was employed to perform all-against-all comparisons using BLASTP. All protein sequences were compared against a database containing protein data sets for all the species under an $E$ value of $1 \times 10^{-5}$. The BLASTP results were further filtered if the aligned region length was $<50 \%$ of any of the aligned two protein sequences. The Markov cluster (MCL) algorithm was then used to cluster the BLASTP results into groups of homologous proteins with an inflation parameter of 1.5 . To analyze the evolution of gene families, CAFE was used to calculate the number of expansions and contractions of gene families ${ }^{41}$.

\section{Phylogenetic analysis}

To build the phylogenetic tree, redundant sequences (90\% identity or more) from the same organism were removed using CD-HIT ${ }^{42}$. Then, homolog clusters were predicted by comparing each pair of the nine plant genomes (A. graveolens, D. carota, M. guttatus, S. tuberosum, S. lycopersicum, Oryza sativa, Coffea canephora, Actinidia chinensis, and A. thaliana) using OrthoMCL. The protein sequences for clusters containing a single-copy gene of each species were aligned with MUSCLE v3.8.31 ${ }^{43}$. Then, positions showing poor alignment were eliminated with $G$ blocks (version 0.91b). All alignments were subsequently concatenated to one super alignment. The phylogeny was reconstructed by the neighbor-joining method using the Jones-Taylor-Thornton model ${ }^{44}$. The reliability of the tree topology was measured by bootstrapping (1000 replications). Evolutionary analysis was conducted in MEGA $7^{45}$. The species divergence time in the phylogenetic tree was estimated via the Bayesian relaxed molecular clock (BRMC) method using the program MULTIDIVTIME, which was implemented in the Thornian Time Traveller (T3) package ${ }^{46,47}$. To estimate the synonymous substitutions per synonymous site $(K s)$, all paralogous gene pairs were analyzed with the maximum likelihood method in the PAML program ${ }^{48}$.

\section{Transcription factors}

Reference information was collected from PlnTFDB ${ }^{49}$, an integrated plant transcription factor database including genes from A. thaliana, Populus trichocarpa, and Oryza sativa (http://plntfdb.bio.uni-potsdam.de). For each transcription factor family, conserved domains were used as queries for searching similar sequences in the celery genome. The protein domains of the identified transcription factors were classified using the Pfam database.

\section{Functional genes}

Celery resistance-related genes were identified based on the most conserved motif structures of plant resistance proteins, including coiled coil (CC), Toll/Interleukin-1 receptor (TIR), nucleotide-binding site (NBS), and leucine-rich repeat (LRR) finger domains. Conserved motifs were derived from domain profiles retrieved from the PFAM, PANTHER, PRINTS, PROSITE, SMART, and SUPERFAMILY databases. PAIRCOIL2 was used to specifically detect $\mathrm{CC}$ domains.

To identify the genes involved in the flavonoid biosynthesis pathway in celery, the assembled genes were annotated with the corresponding Enzyme Commission numbers against the KEGG database. All candidate genes 
were further submitted to the NCBI database to obtain gene function information. Terpenoid synthase (TPS) proteins were identified by screening the celery genome sequences using HMMER3.0 software with domain models PF03936 and PF01397 as queries ${ }^{50}$.

\section{Data access}

The whole-genome sequences of celery have been deposited at CeleryDB under accession version $1.0^{51}$. The genome data can be accessed at http://apiaceae.njau.edu. $\mathrm{cn} /$ celerydb.

\section{Results}

\section{Genome sequencing and assembly}

For celery genome sequencing, a total of 68 genomic libraries with three small insert sizes (180 bp, $500 \mathrm{bp}$, and $800 \mathrm{bp}$ ) and three large insert sizes ( $2 \mathrm{~kb}, 5 \mathrm{~kb}$, and $10 \mathrm{~kb})$ were prepared. We generated raw sequences from the next-generation sequencing platform HiSeq 2000. After filtering, a total of $600.8 \mathrm{~Gb}$ of clean data were obtained from the paired-end libraries with different insert sizes (Supplementary Table S1). The genome size was estimated to be $3.18 \mathrm{~Gb}$ based on the 17 -mer depth distribution (Supplementary Fig. S1 and Table S2). All clean data were assembled into contigs and scaffolds using SOAP de novo, resulting in a final assembly of $2.21 \mathrm{~Gb}$ with N50 sizes of 13,108 bp for contigs and 35,567 bp for scaffolds (Tables 1 and 2). The total clean data generated represented $188.93 \times$ coverage of the estimated celery genome, and our assembly accounted for $\sim 70 \%$ of the estimated genome. The assembled genome exhibited highly complete Benchmarking Universal Single-Copy Orthologs (BUSCO) (90.8\%) (Supplementary Table S3). Compared with another Apiaceae species, carrot, the genome size of celery is much greater. The percentage of the GC content in celery genome was $35.35 \%$, which was close to those in the genomes of carrot (34.80\%),

Table 1 Statistics of the celery genome assembly.

\begin{tabular}{ll}
\hline Feature & Value \\
\hline Genome size & $2.21 \mathrm{~Gb}$ \\
Genome GC\% & $35.35 \%$ \\
Gene number & 34,277 \\
Gene no. per $100 \mathrm{~kb}$ & 1.44 \\
Average gene length (bp) & 3267 \\
Exon region GC (\%) & $42.06 \%$ \\
Exon number & 180,591 \\
Average exon length (bp) & 243.48 \\
Exon no. per gene & 5.27 \\
\hline
\end{tabular}

Arabidopsis (36.06\%), and tomato (34.05\%) but lower than those in tea tree $(42.31 \%)$ and rice $(43.57 \%)$ (Supplementary Table S4).

\section{Repetitive sequence analysis}

De novo repeat identification using RepeatMasker and homology analysis against the RepBase library showed that repetitive DNA (excluding low-complexity sequences) accounted for $68.88 \%$ of the genome. The classification of the observed transposable elements into known classes revealed that the majority of repetitive sequences were LTR retrotransposons (44.07\%), whereas $3.30 \%$ and $2.76 \%$ of the repeat element types were DNA transposons and simple repeat elements, respectively (Table 3).

The fraction of repetitive sequences in the celery genome $(68.88 \%)$ was higher than that in the carrot (45.95\%) and physic nut genomes $(49.81 \%)$ but lower than that detected in the genomes of tea tree $(80.89 \%)$ and ginkgo (76.58\%) (Supplementary Table S5). In comparison, celery, tea tree, and ginkgo exhibit larger genome sizes than carrot and physic nut. In addition, LTRs occupied the absolute dominant position among the repetitive sequences of these five plants. In this study, the activity of LTRs at the molecular level was analyzed. The amplification of celery LTR elements was relatively active $\sim 2.5 \sim 4$ Mya (million years ago) and $5 \mathrm{Mya}$, which was close to the recent whole-genome duplication (WGD) event that occurred in celery 1.9 Mya (Supplementary Fig. S2). Previous studies on sequenced plant genomes have shown that the proportion of repetitive sequences is one of most important factors affecting genome size ${ }^{52-54}$. The recently inserted LTRs may be an important factor affecting the size of the celery genome.

\section{Gene prediction and annotation}

To predict protein-coding genes in the celery genome, de novo gene prediction programs and homology-based methods were combined to assemble the results. We

Table 2 Statistics of the assembly size of the contigs and scaffolds of celery.

\begin{tabular}{lll}
\hline Property & Contig & Scaffold \\
\hline Min sequence length (bp) & 500 & 500 \\
Max sequence length (bp) & 228,328 & 556,749 \\
Total sequence number & 432,762 & 257,842 \\
N50 length (bp) & 13,108 & 35,567 \\
N90 length (bp) & 1136 & 4841 \\
N number & 648,982 & $280,637,212$ \\
N rate (\%) & 0.031 & 11.8 \\
Total sequence length (bp) & $2,017,581,028$ & $2,372,941,895$ \\
\hline
\end{tabular}


Table 3 Repeat element analysis in the celery genome.

\begin{tabular}{llll}
\hline Repeat elements & $\begin{array}{l}\text { Copies } \\
\text { (numbers) }\end{array}$ & $\begin{array}{l}\text { Repeat } \\
\text { size (bp) }\end{array}$ & $\begin{array}{l}\text { Percentage of the } \\
\text { assembled } \\
\text { genome (\%) }\end{array}$ \\
\hline DNA transposon & 157,948 & $78,298,900$ & 3.30 \\
LINE & 42,679 & $34,853,999$ & 1.47 \\
Low complexity & 174,767 & $9,739,053$ & 0.41 \\
LTR/Copia & 573,134 & $481,821,466$ & 20.30 \\
LTR/Gypsy & 605,914 & $519,802,870$ & 21.91 \\
LTR/others & 58,772 & $44,248,275$ & 1.86 \\
SINE & 4272 & 454,864 & 0.02 \\
Satellite & 7993 & $8,983,101$ & 0.38 \\
Simple repeat & 860,188 & $65,493,490$ & 2.76 \\
Unknown & 453,513 & $390,743,993$ & 16.47 \\
Total & $2,939,180$ & $1,634,440,011$ & 68.88 \\
\hline
\end{tabular}

Table 4 Number of noncoding RNAs in the celery genome.

\begin{tabular}{lllll}
\hline Type & Number & Size (bp) & $\begin{array}{l}\text { Average } \\
\text { length (bp) }\end{array}$ & $\begin{array}{l}\text { Percentage of } \\
\text { genome (\%) }\end{array}$ \\
\hline miRNA & 116 & 14,759 & 127.23 & 0.00062 \\
tRNA & 891 & 67,306 & 75.54 & 0.00284 \\
rRNA & 374 & 321,240 & 858.93 & 0.01354 \\
SnRNA & 8,878 & 938,847 & 105.75 & 0.00396 \\
\hline
\end{tabular}

predicted 34,277 genes with an average length of $3267 \mathrm{bp}$ and a mean of 5.27 exons per gene in the celery genome (Table 1 and Supplementary S4). Compared with other species, the celery gene number was similar to that of tomato $(34,727)$, larger than those of Arabidopsis $(27,416)$ and carrot $(32,113)$, and lower than those of tea tree $(36,951)$ and rice $(57,939)$ (Supplementary Table S4). In addition to protein-coding genes, we identified 891 tRNA, 116 miRNA, 374 rRNA, and 8878 snRNA genes in the celery genome, which constituted $\sim 0.021 \%$ of the genome sequences (Table 4).

A sequence similarity search was performed against public databases to investigate putative functions. A total of 34,143 genes were annotated using the $\mathrm{Nr}$, InterPro, GO, and KEGG databases (Supplementary Table S6). Based on the GO database, 16,920 genes were annotated to three gene ontology classes: biological process, cellular component, and molecular function, with 1223 functional terms (Fig. S3). The most-frequent functional clusters in the celery genome were protein binding, ATP binding and oxidation-reduction processes. By mapping to the KEGG database, 9463 genes were assigned to KEGG metabolic pathways.

Based on the pair-wise protein sequence similarities, we carried out a gene family analysis of celery genes. A total of 27,549 genes were clustered in 15,164 gene families with an average size of 1.82, and 6728 of these genes did not exhibit homologous sequences (Supplementary Table S7). The number of members in the gene families varied greatly, and group 4 exhibited the largest number, including 201 genes (Supplementary Fig. S4). The gene ontology category analysis showed that the gene families that contained the most members were widely involved in biological processes such as cell recognition, serine-type endopeptidase activity, flavin adenine dinucleotide binding, protein phosphorylation, and zinc ion binding (Supplementary Table S8). For the analysis of the specific and shared gene families among species, conserved putative genes from five plants were used to identify gene family clusters. Among the total 81,793 clusters, 9442 clusters were observed in all investigated species, and 12,283 appeared to be lineage specific to celery, whereas 13,934 were shared with $D$. carota, 10,224 with A. thaliana, 11,168 with S. lycopersicum, and 10,940 with M. guttatus (Fig. 2a). The celery-specific genes were analyzed on the basis of the results of gene prediction and annotation (Supplementary Table S9). A total of 783 celery-specific genes corresponded to 1456 annotation results, which were divided into $155 \mathrm{GO}$ terms.

\section{Celery-specific transcription factors and disease resistance- related genes}

Within the celery genome, the percentage of transcription factors $(4.95 \%)$ was slightly greater than that in the rice genome $(4.80 \%)$ but lower than those in carrot (8.41\%), Arabidopsis (7.00\%), and tomato (7.09\%). In the celery genome, there are six transcription factor families (FAR1, ERF, MYB, MYB-related, bHLH, and NAC) that contain $>100$ members. A total of 184 celery-specific transcription factors were identified from the 1698 putative transcription factors of celery, which were classified into 12 transcription factor families (Fig. S5). Similar to the percentage of total transcription factors in the celery genome, the FAR1 (59\%) family yielded the largest number of celery-specific transcription factors. NAC was the second largest family of celery-specific transcription factors, with 28 members. The number of celery-specific transcription factors in both the MYB family and the MYB-related family was 10 .

Celery encounters various forms of environmental stress, including a wide range of pests and pathogens that negatively affect celery growth and yield. A variety of disease resistance-related genes are induced to respond to stresses and to increase celery tolerance. NBS and carboxy-terminal LRR domains are found in the majority 


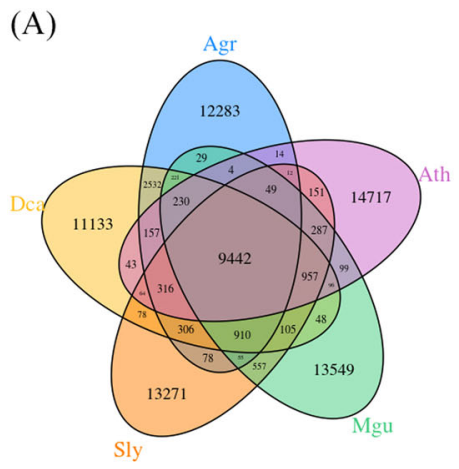

(C)

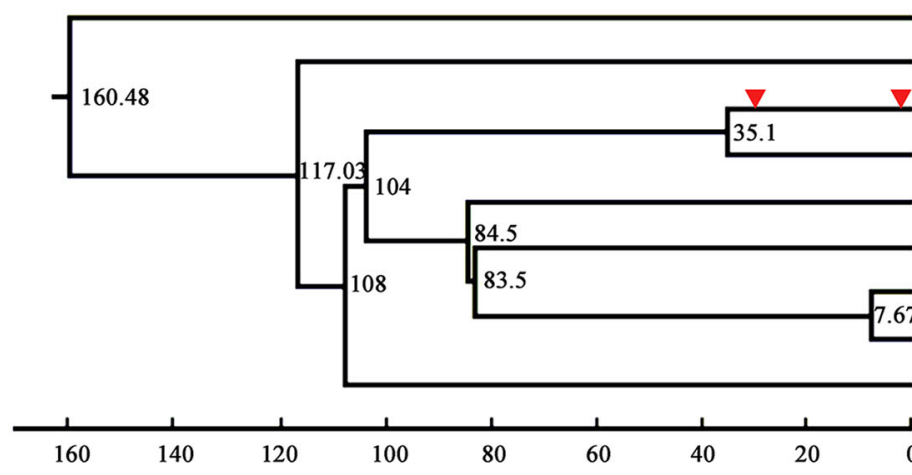

(B)

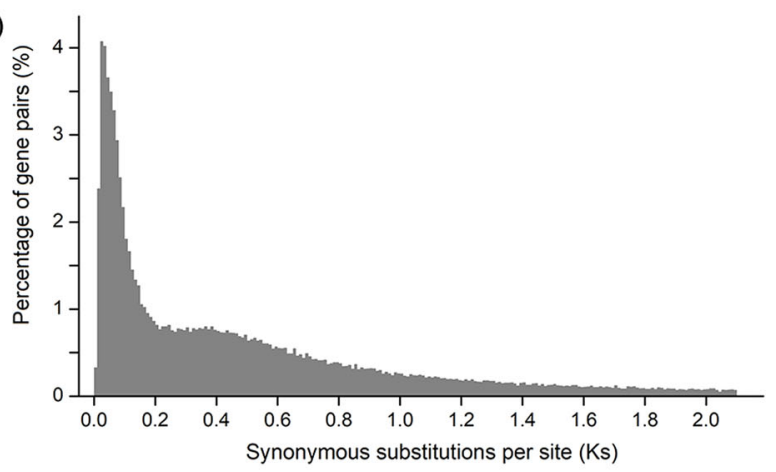

Gene families

Expansion/Contraction

O. sativa

A. thaliana

A. graveolens

D. carota

C. canephora

M. guttatus

S. tuberosum

S. lycopersicum

A. chinensis
- $+3915 /-16649$

D $+2961 /-15033$

$+2131 /-2721$

(1) $+2342 /-2043$

(C) $+1408 /-8222$

(1) $+2518 /-7305$

(1) $+2016 /-2444$

$+1620 /-1849$

$+5384 /-12946$

Fig. 2 Comparative genomic and phylogenetic relationship analyses. a Venn diagram showing the cluster distribution of shared gene families among A. graveolens (Agr), M. guttatus (Mgu), S. lycopersicum (Sly), D. carota (Dca), and A. thaliana (Ath). b Ks distribution of paralogous gene pairs in the celery genome. The probability density of Ks was estimated using the "density" function in the R language. $\mathbf{c}$ Evolutionary analysis of celery and eight other plant species. The divergence time was estimated with the calibration time for S. tubersum and S. lycopersicum. The pie charts show the proportions of expanded (green), contracted (red), and unchanged (blue) gene families. The potential WGD events of celery are indicated with red triangles.

of $\mathrm{R}$ proteins ${ }^{55,56}$. A total of 201 NBS-containing resistance genes were identified based on resistance domain analyses in the celery genome, all of which were further classified into six groups: TIR-NBS-LRR, CC-NBS-LRR, CC-NBS, TIR-NBS, NBS-LRR, and NBS (Table 5). The total number of NBS-LRR genes was 146 in $\operatorname{carrot}^{20}, 176$ in Arabidopsis ${ }^{57}, 107$ in maize ${ }^{58}$, and 472 in rice ${ }^{59}$. Among these genes in the celery genome, one class, NBS, presented a markedly greater number in celery (126 genes) than in other plants including carrot (19), Arabidopsis (1), maize (7) and rice (25). The NBS-LRR genes present in monocotyledons and dicotyledons showed different characteristics. The CNL and NL groups accounted for a large proportion of the genes in monocotyledonous plants (maize and rice), whereas no genes encoding a TIR domain were found. However, all of the groups existed in dicotyledonous plants, but their distributions were different among species.

\section{Evolution of celery}

WGD events occur widely in flowering plants and are one of the most important drivers of genome evolution,
Table 5 Comparison of the numbers and classifications of genes encoding an NBS domain in celery, carrot, Arabidopsis, maize, and rice.

\begin{tabular}{lllllll}
\hline $\begin{array}{l}\text { Protein } \\
\text { domains }\end{array}$ & Letter code & Celery & Carrot & Arabidopsis & Maize & Rice \\
\hline $\begin{array}{l}\text { CC-NBS- } \\
\text { LRR }\end{array}$ & CNL & 6 & 57 & 51 & 58 & 160 \\
$\begin{array}{l}\text { TIR-NBS- } \\
\text { LRR }\end{array}$ & TNL & 0 & 4 & 92 & 0 & 0 \\
CC-NBS & CN & 47 & 0 & 5 & 11 & 7 \\
TIR-NBS & TN & 19 & 0 & 21 & 0 & 0 \\
NBS-LRR & NL & 3 & 66 & 6 & 31 & 280 \\
NBS & $N$ & 126 & 19 & 1 & 7 & 25 \\
Total & & 201 & 146 & 176 & 107 & 472 \\
\hline
\end{tabular}

the origination of new species, and gene neofunctionalization $^{54,60}$. Ks values can be used to estimate the timing of large-scale duplications ${ }^{61}$. The distribution of $K s$ values 
between celery paralogous pairs displayed two peaks, at 0.025 and 0.385 (Fig. 2b), which indicated recent WGD events in the evolution of celery. Based on the generally accepted evolutionary rate ${ }^{62}$, the WGD events might have occurred at times of $\sim 1.9$ and $29.6 \mathrm{Mya}$.

Using the 28,874 orthologous gene families identified by OrthoMCL from the celery genome and eight other fully sequenced genomes, we constructed a phylogenetic tree to show the relationships among the nine higher plants. The times of divergence among these plant species were also estimated (Fig. 2c). We used the time of divergence between S. tubersum and S. lycopersicum as a calibration point. Celery and carrot diverged from a common ancestor $~ 35.1$ Mya. In addition, we performed a comparative analysis of gene family evolution in the nine plants in the phylogenetic tree. A total of 2131 gene families were expanded in the celery lineage, whereas 2721 gene families had undergone contraction.

\section{Apigenin biosynthesis pathway in celery}

Apigenin is one of the most important flavonoid compounds and exhibits a variety of biological activities and pharmacological effects ${ }^{3}$. Compared with other plants, celery has a higher content of apigenin ${ }^{6,8}$. In light of the flavonoid compounds among the important secondary metabolites of celery, we analyzed the genes involved in the flavonoid biosynthesis pathway. Most of these flavonoid biosynthesis pathway genes were found in our dataset (Supplementary Fig. S6). Here, 41 genes that were putatively involved in the flavonoid biosynthesis pathway, mainly encoding 13 different enzymes, were identified in celery based on the genome sequences (Supplementary Table S10). Compared with the flavonoid biosynthesis genes of Arabidopsis, rice, and tomato (Supplementary Tables S10 and S11), the greatest number of genes was found in tomato (49), followed by celery (41), rice (34), and Arabidopsis (21). The main flavonoid genes in tomato are the $H C T$ and CCOAOMT genes, which account for $55 \%$ of these genes, whereas the numbers of different genes in other species are relatively diverse.

Chalcone synthase (CHS) is a key enzyme in the apigenin biosynthesis pathway, and more genes encoding CHS were found in celery than in other plants. One chalcone isomerase $(\mathrm{CHI})$ was obtained from the celery genome. Flavone synthase I (FNSI) is another enzyme that is necessary for apigenin biosynthesis. Two FNSI genes were detected in celery but not in Arabidopsis, rice, or tomato. Based on celery RNA-seq data from three developmental stages ${ }^{63,64}$, the expression changes in apigenin-related genes were analyzed, and the results were presented in a heatmap. Most of the apigenin biosynthesis-related genes showed the highest expression levels at early stages, and their expression trends decreased with celery growth and development (Fig. 3).

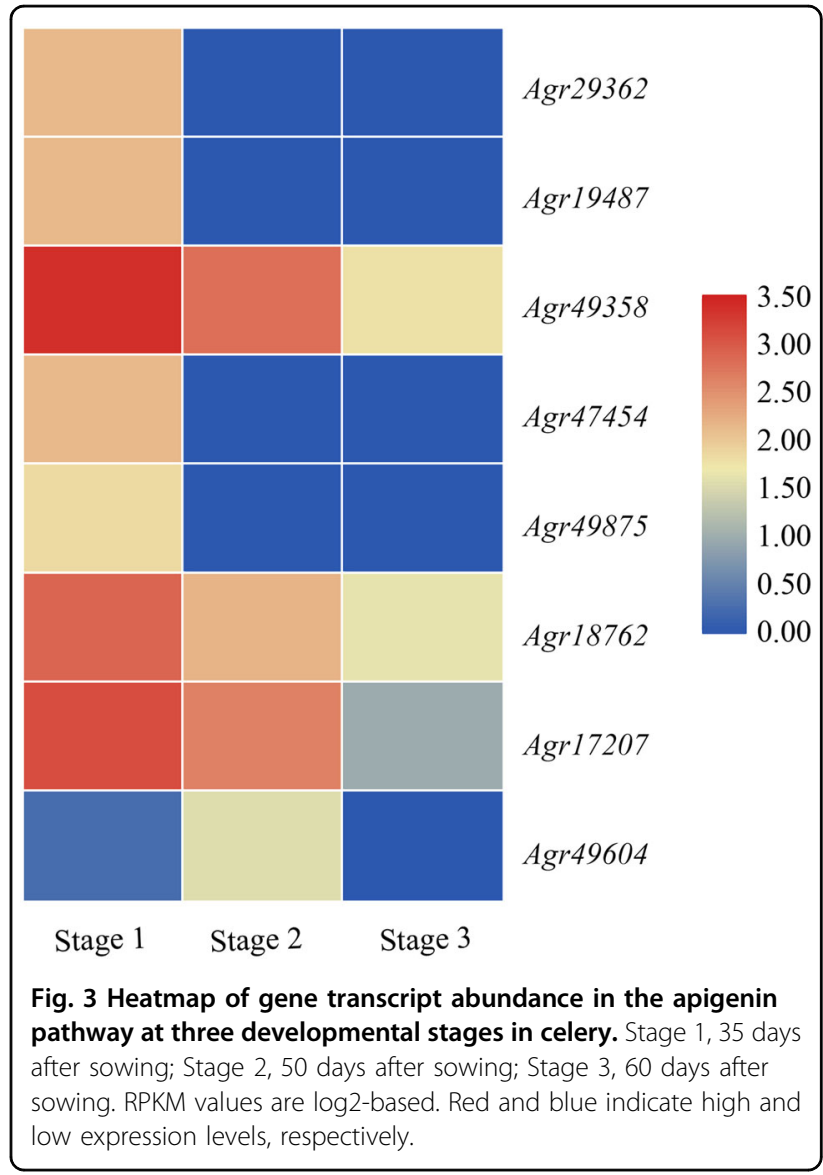

This work extends previous reports on related genes involved in apigenin biosynthesis and provides an overview of apigenin biosynthesis pathways in celery.

\section{Terpenoid synthase family genes}

The flavor of celery is mainly attributed to its terpenoid content. TPSs are the key enzymes that catalyze complex multiple-step cyclization in terpene metabolism. A total of 38 putative TPS proteins were screened in the celery genome by using HMMER software based on the highly conserved domain. To confirm the classification of TPS family proteins in celery, we constructed a phylogenetic tree by aligning the TPS proteins among celery, carrot ${ }^{20}$, Arabidopsis $^{65}$, and tomato ${ }^{66}$. All the proteins were divided into five subfamilies: TPS-a, TPS-b, TPS-c, TPS-e/f, and TPS-g (Fig. 4a). In angiosperms, the TPS-b and TPS-a subfamilies account for most of the sesquiterpene synthases and monoterpene synthases, which constitute the majority of TPS proteins. DCAR_023152 (DcTPS1) and DCAR_012963 (DcTPS2) were two TPSs found in carrot belonging to the TPS-a subfamily and TPS-b subfamily, respectively ${ }^{67}$. The TPSs of celery showed the closest phylogenetic relationships with those of carrot, another Apiaceae family plant. In the celery genome, the 


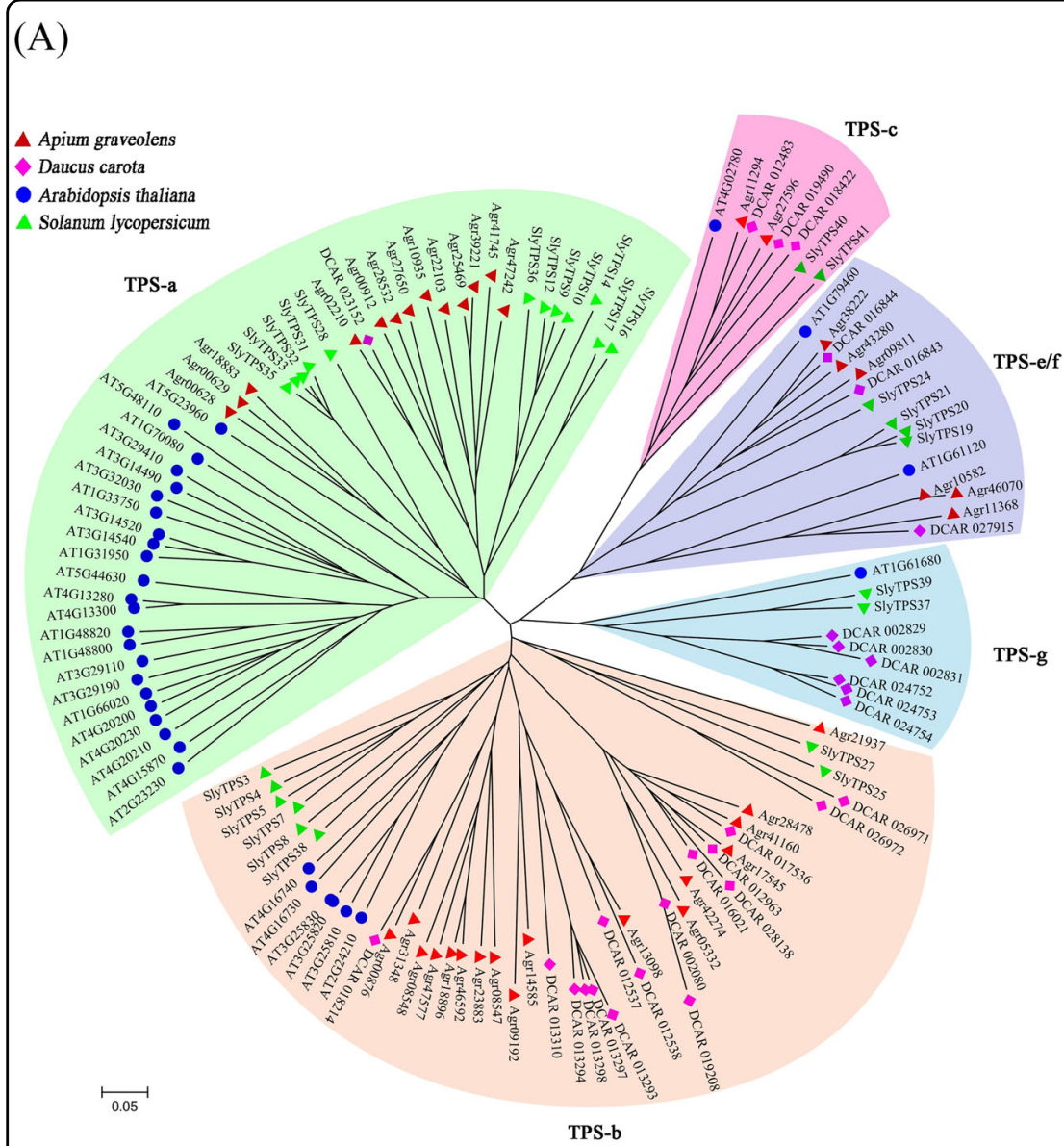

(B)

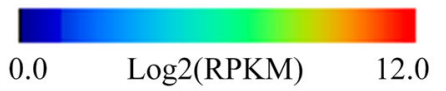

Stage 1 Stage 2 Stage 3

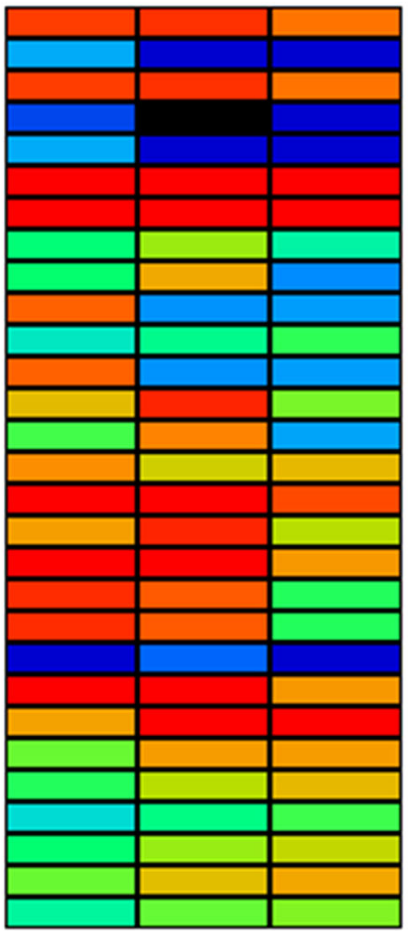

Agr 47242
Agr41745
Agr25469
Agr22103
Agr10935
Agr27650
Agr28532
Agr02210
Agr00912
Agr18883
Agr00629
Agr00628
Agr13098
Agr14585
Agr21937
Agr28478
Agr41160
Agr08547
Agr46592
Agr18896
Agr08548
Agr31348
Agr11294
Agr27596
Agr38222
Agr43280
Agr09811
Agr10582
Agr11368

Fig. 4 Phylogenetic tree and heatmap of TPS proteins in celery. a Phylogenetic tree of all TPS proteins among celery, carrot, Arabidopsis, and tomato. $\mathbf{b}$ Heatmap clustering of TPS gene transcript abundances at three developmental stages (Stage 1, 35 days after sowing; Stage 2, 50 days after sowing; Stage 3, 60 days after sowing) in celery. The RPKM values are log2-based. Red and black indicate high and low expression levels, respectively.

TPS-b and TPS-a subfamilies contained 17 and 13 members, and only six and two proteins belonged to the TPS-e/f and TPS-c subfamilies, respectively, whereas no proteins clustered within the TPS-g subfamily in celery. Based on previous transcriptome data from celery ${ }^{63,64}$, we further analyzed the expression abundance of TPS genes in three developmental stages. As illustrated in Fig. 4b, 30 TPS genes were continuously expressed during development. Compared with stage 3, most TPS genes exhibited higher transcript abundance in stage 1 and stage 2 , suggesting that these genes may be involved in a variety of terpenoid metabolic processes during celery growth and development.

\section{Discussion}

Next-generation sequencing is a powerful method for genome research. With the continuous development of sequencing technology, whole-genome sequencing has been carried out in increasing numbers of species, providing a great deal of biological information for genome mapping, functional gene mining and molecular breeding. Celery is normally classified as a cool-season vegetable and widely consumed around the world, and the cultivation history of celery is longer than 2000 years $^{2,3}$. To understand the genetic system and evolution of Apiaceae, we sequenced the genome of celery, which offers new insights and provides resources for molecular breeding.

Here, we report the first genome data for celery. The genome size was estimated to be $3.18 \mathrm{~Gb}$, which is close to the previous size estimate obtained using flow cytometry of $\sim 3.14 \mathrm{~Gb}$. The genome size of celery is far larger than that of carrot, another Apiaceae species, and is even larger than those of some angiosperm plants, such as Arabidopsis, rice and poplar. Celery is one of the sequenced species with the largest genome sizes among those sequenced in recent years, and the celery genome is mainly composed of repetitive sequences, which account for $68.88 \%$ of the genome, whereas this percentage is $45.95 \%$ in carrot. By comparing the genome components of species with different genome sizes, we found that the 
proportions of repetitive sequences were obviously higher in species with large genome sizes than in other species (e.g., $80.89 \%$ in tea tree ${ }^{68}$ and $76.58 \%$ in ginkgo ${ }^{69}$ ).

WGD events and tandem duplications are the most important determinants of the variation in the genome sizes of angiosperms ${ }^{54,70}$. As two important crops of the Apiaceae family, the evolutionary analysis suggested that celery and carrot may have diverged $\sim 35.1$ Mya. In addition, a recent WGD event was deduced to have occurred in celery 1.9 Mya. This genome duplication event not only caused genome expansion in celery, making its genome larger than of carrot, but may also have contributed to the physiological and morphological diversity of the celery lineage.

As a medicinal and edible plant, celery contains various biologically active substances. The biosynthesis and metabolism pathways of these active substances are a major research focus. The genome sequencing data provide new insights into the unique biosynthetic processes of celery, particularly for some important secondary metabolite pathways. Based on the genome sequences, a total of 41 genes that mainly encoded the enzymes in the flavonoid biosynthesis pathway were screened out. Compared with Arabidopsis, rice and tomato, the celery genome contains the richest set of apigenin biosynthesis genes, including $C H S, F 3 H, F 3^{\prime} H$, and FNSI. The duplication and evolution of these genes in celery might be important contributors to enhancing the ability of celery to synthesize flavonoids.

The rich flavor of celery is mainly owing to its high content of terpenoids. In addition, terpenoids play numerous roles in plants, such as functions in resistance to pathogens and pests, acting as the precursors of plant hormones, and participating in plant growth and development ${ }^{71-73}$. The obtained genome sequences helped us to identify TPS family genes, which are essential for exploring terpene synthases and terpenoid metabolism. Expression abundance analysis showed that most of the TPS genes were strongly expressed in the late developmental stage of celery, which was consistent with the increased expression of TPS genes during carrot maturation ${ }^{67}$. Although further research is needed to confirm the regulatory mechanisms of TPS genes, the results reveal that TPS genes show temporal specificity during different developmental periods. The information and genome sequence resources reported for the celery genome in this study can enhance both fundamental and applied research on celery and other Apiaceae family plants.

\section{Acknowledgements}

The research was supported by the Jiangsu Agriculture Science and Technology Innovation Fund (CX(18)2007), New Century Excellent Talents in University (NCET-11-0670), National Natural Science Foundation of China
(31272175), Jiangsu Natural Science Foundation (BK20130027), Priority Academic Program Development of Jiangsu Higher Education Institutions (PAPD), and Jiangsu Shuangchuang Project.

Conflict of interest

The authors declare that they have no conflict of interest.

Supplementary Information accompanies this paper at (https://doi.org/ 10.1038/s41438-019-0235-2).

Received: 11 May 2019 Revised: 2 November 2019 Accepted: 5 December 2019

Published online: 06 January 2020

\section{References}

1. Burt, S. Essential oils: their antibacterial properties and potential applications in foods - a review. Int. J. Food Microbiol. 94, 223-253 (2004).

2. Al-Asmari, A. K., Athar, M. T. \& Kadasah, S. G. An updated phytopharmacological review on medicinal plant of Arab region: Apium graveolens Linn. Pharmacogn. Rev. 11, 13-18 (2017).

3. Li, M. Y. et al. Advances in the research of celery, an important Apiaceae vegetable crop. Crit. Rev. Biotechnol. 38, 172-183 (2018).

4. Li, J. W., Ma, J., Feng, K., Xu, Z. S. \& Xiong, A. S. Transcriptome profiling of $\beta$-carotene biosynthesis genes and $\beta$-carotene accumulation in leaf blades and petioles of celery cv. Jinnanshiqin. Acta Biochem. Biophys. Sin. 51, 116-119 (2019).

5. Lin, L. Z., Lu, S. M. \& Harnly, J. M. Detection and quantification of glycosylated flavonoid malonates in celery, Chinese celery, and celery seed by LC-DAD-ESI/ MS. J. Agric. Food Chem. 55, 1321-1326 (2007).

6. Hertog, M. G. L., Hollman, P. C. H. \& Venema, D. P. Optimization of a quantitative HPLC determination of potentially anticarcinogenic flavonoids in vegetables and fruits. J. Agric. Food Chem. 40, 1591-1598 (1992).

7. Feng, $\mathrm{K}$. et al. AgMYB2 transcription factor is involved in the regulation of anthocyanin biosynthesis in purple celery (Apium graveolens L.). Planta 248, 1249-1261 (2018).

8. Tan, G. F., Ma, J., Zhang, X. Y., Xu, Z. S. \& Xiong, A. S. AgFNS overexpression increase apigenin and decrease anthocyanins in petioles of transgenic celery. Plant Sci. 263, 31-38 (2017)

9. Funakoshi-Tago, M., Nakamura, K, Tago, K, Mashino, T. \& Kasahara, T. Antiinflammatory activity of structurally related flavonoids, Apigenin, Luteolin and Fisetin. Int. Immunopharmacol. 11, 1150-1159 (2011).

10. Huang, C. S. et al. Protection by chrysin, apigenin, and luteolin against oxidative stress is mediated by the Nrf2-dependent up-regulation of heme oxygenase 1 and glutamate cysteine ligase in rat primary hepatocytes. Arch. Toxicol. 87, 167-178 (2013)

11. Momin, R. A. \& Nair, M. G. Mosquitocidal, nematicidal, and antifungal compounds from Apium graveolens L. seeds. J. Agric. Food Chem. $\mathbf{4 9}$ 142-145 (2001).

12. Tang, D., Chen, K. L., Huang, L. Q. \& Li, J. Pharmacokinetic properties and drug interactions of apigenin, a natural flavone. Expert Opin. Drug Metab. Toxicol. 13, 323-330 (2017).

13. Gadermaier, G. et al. Molecular characterization of Api g 2, a novel allergenic member of the lipid-transfer protein 1 family from celery stalks. Mol. Nutr. Food Res. 55, 568-577 (2011).

14. Feng, K. et al. Isolation, purification, and characterization of AgUCGalT1, a galactosyltransferase involved in anthocyanin galactosylation in purple celery (Apium graveolens L.). Planta 247, 1363-1375 (2018).

15. Kun, L. I. \& Zhang, D. C. \& Shan, You-Xi. The quantitation of flavonoids in leaf and stalk of different celery cultivars and the correlation with antioxidation activity. Acta Horticulturae Sin. 57, 133-139 (2011).

16. Arabidopsis Genome, I. Analysis of the genome sequence of the flowering plant Arabidopsis thaliana. Nature 408, 796-815 (2000).

17. Velasco, R. et al. The genome of the domesticated apple (Malus $\times$ domestica Borkh.). Nat. Genet. 42, 833-839 (2010).

18. Schnable, P. S. et al. The B73 maize genome: complexity, diversity, and dynamics. Science 326, 1112-1115 (2009).

19. Huang, S. et al. The genome of the cucumber, Cucumis sativus L. Nat. Genet. 41, 1275-1281 (2009). 
20. Iorizzo, M. et al. A high-quality carrot genome assembly provides new insights into carotenoid accumulation and asterid genome evolution. Nat. Genet. $\mathbf{4 8}$ 657-666 (2016).

21. Sun, D. L. et al. Draft genome sequence of cauliflower (Brassica oleracea L. var. botrytis) provides new insights into the $C$ genome in Brassica species. Hortic. Res. 6, 82 (2019)

22. Xu, Z. S., Tan, H. W., Wang, F., Hou, X. L. \& Xiong, A. S. CarrotDB: a genomic and transcriptomic database for carrot. Database 2014, bau096 (2014).

23. Rogers, S. O. \& Bendich, A. J. Extraction of DNA from milligram amounts of fresh, herbarium and mummified plant tissues. Plant Mol. Biol. 5, 69-76 (1985).

24. Martin, M. Cutadapt removes adapter sequences from high-throughput sequencing reads. Embnet J. 17, 1 (2011).

25. Luo, R. et al. SOAPdenovo2: an empirically improved memory-efficient shortread de novo assembler. Gigascience 1, 18 (2012).

26. Simao, F. A., Waterhouse, R. M., loannidis, P., Kriventseva, E. V. \& Zdobnov, E. M. BUSCO: assessing genome assembly and annotation completeness with single-copy orthologs. Bioinformatics 31, 3210-3212 (2015).

27. Stanke, M. et al. AUGUSTUS: ab initio prediction of alternative transcripts. Nucleic Acids Res. 34, W435-439 (2006).

28. Korf, I. Gene finding in novel genomes. BMC Bioinformatics 5, 59 (2004).

29. $\mathrm{Xu}, \mathrm{X}$. et al. Genome sequence and analysis of the tuber crop potato. Nature 475, 189-U194 (2011).

30. Slater, G. S. \& Birney, E. Automated generation of heuristics for biological sequence comparison. BMC Bioinformatics 6, 31 (2005).

31. Ashburner, M. et al. Gene ontology: tool for the unification of biology. The Gene Ontology Consortium. Nat. Genet. 25, 25-29 (2000).

32. Tatusov, R. L. et al. The COG database: an updated version includes eukaryotes. BMC Bioinformatics 4, 41 (2003).

33. Conesa, A. et al. Blast2GO: a universal tool for annotation, visualization and analysis in functional genomics research. Bioinformatics 21, 3674-3676 (2005)

34. Ye, J. et al. WEGO: a web tool for plotting GO annotations. Nucleic Acids Res. 34, W293-297 (2006).

35. Lowe, T. M. \& Eddy, S. R. tRNAscan-SE: a program for improved detection of transfer RNA genes in genomic sequence. Nucleic Acids Res. 25, 955-964 (1997).

36. Nawrocki, E. P., Kolbe, D. L. \& Eddy, S. R. Infernal 1.0: inference of RNA alignments. Bioinformatics 25, 1335-1337 (2009).

37. Nawrocki, E. P. et al. Rfam 12.0: updates to the RNA families database. Nucleic Acids Res. 43, D130-D137 (2015).

38. Chen, N. Using RepeatMasker to identify repetitive elements in genomic sequences. Curr. Protoc. Bioinformatics 25, 4.10.1-4.10.14 (2004).

39. Li, L., Stoeckert, C. J. \& Roos, D. S. OrthoMCL: identification of ortholog groups for eukaryotic genomes. Genome Res. 13, 2178-2189 (2003).

40. Goodstein, D. M. et al. Phytozome: a comparative platform for green plant genomics. Nucleic Acids Res. 40, D1178-1186 (2012).

41. De Bie, T., Cristianini, N., Demuth, J. P. \& Hahn, M. W. CAFE: a computational tool for the study of gene family evolution. Bioinformatics 22, 1269-1271 (2006).

42. Fu, L. M., Niu, B. F., Zhu, Z. W., Wu, S. T. \& Li, W. Z. CD-HIT: accelerated for clustering the next-generation sequencing data. Bioinformatics $\mathbf{2 8}, 3150-3152$ (2012).

43. Edgar, R. C. MUSCLE: multiple sequence alignment with high accuracy and high throughput. Nucleic Acids Res. 32, 1792-1797 (2004)

44. Jones, D. T., Taylor, W. R. \& Thornton, J. M. The rapid generation of mutation data matrices from protein sequences. Comput. Appl. Biosci. 8, 275-282 (1992).

45. Kumar, S., Stecher, G. \& Tamura, K. MEGA7: Molecular evolutionary genetics analysis version 7.0 forbigger datasets. Mol. Biol. Evol. 33, 1870-1874 (2016).

46. Wikstrom, N., Savolainen, V. \& Chase, M. W. Evolution of the angiosperms: calibrating the family tree. Proc. Biol. Sci. 268, 2211-2220 (2001).

47. Crepet, W. L., Nixon, K. C. \& Gandolfo, M. A. Fossil evidence and phylogeny: The age of major angiosperm clades based on mesofossil and macrofossil evidence from cretaceous deposits. Am. J. Bot. 91, 1666-1682 (2004).

48. Yang, Z. PAML: a program package for phylogenetic analysis by maximum likelihood. Comput. Appl. Biosci. 13, 555-556 (1997).
49. Riano-Pachon, D. M., Ruzicic, S., Dreyer, I. \& Mueller-Roeber, B. PInTFDB: an integrative plant transcription factor database. BMC Bioinformatics 8, 42 (2007).

50. Eddy, S. R. Accelerated profile HMM searches. PLoS Comput Biol. 7, e1002195 (2011).

51. Feng, K. et al. CeleryDB: a genomic database for celery. Database 2018 bay070 (2018).

52. SanMiguel, P. et al. Nested retrotransposons in the intergenic regions of the maize genome. Science 274, 765-768 (1996).

53. Vitte, C. \& Panaud, O. LTR retrotransposons and flowering plant genome size: emergence of the increase/decrease model. Cytogenet. Genome Res. 110, 91-107 (2005).

54. Piegu, B. et al. Doubling genome size without polyploidization: dynamics of retrotransposition-driven genomic expansions in Oryza australiensis, a wild relative of rice. Genome Res. 16, 1262-1269 (2006).

55. DeYoung, B. J. \& Innes, R. W. Plant NBS-LRR proteins in pathogen sensing and host defense. Nat. Immunol. 7, 1243-1249 (2006).

56. Takken, F. L., Albrecht, M. \& Tameling, W. I. Resistance proteins: molecular switches of plant defence. Curr. Opin. Plant Biol. 9, 383-390 (2006).

57. Meyers, B. C., Kozik, A., Griego, A., Kuang, H. \& Michelmore, R. W. Genome-wide analysis of NBS-LRR-encoding genes in Arabidopsis. Plant Cell 15, 809-834 (2003).

58. Cheng, Y. et al. Systematic analysis and comparison of nucleotide-binding site disease resistance genes in maize. FEBS J. 279, 2431-2443 (2012).

59. Zhou, T. et al. Genome-wide identification of NBS genes in japonica rice reveals significant expansion of divergent non-TIR NBS-LRR genes. Mol. Genet. Genomics 271, 402-415 (2004).

60. Van de Peer, Y., Maere, S. \& Meyer, A. The evolutionary significance of ancient genome duplications. Nat. Rev. Genet. 10, 725-732 (2009).

61. Blanc, G. \& Wolfe, K. H. Widespread paleopolyploidy in model plant species inferred from age distributions of duplicate genes. Plant Cell 16, 1667-1678 (2004).

62. Gaut, B. S., Morton, B. R., McCaig, B. C. \& Clegg, M. T. Substitution rate comparisons between grasses and palms: Synonymous rate differences at the nuclear gene Adh parallel rate differences at the plastid gene rbcL. Proc. Natl Acad. Sci. 93, 10274-10279 (1996).

63. Jia, X. L. et al. De novo assembly, transcriptome characterization, lignin accumulation, and anatomic characteristics: novel insights into lignin biosynthesis during celery leaf development. Sci. Rep. 5, 8259 (2015).

64. Li, M. Y., Wang, F., Jiang, Q., Ma, J. \& Xiong, A. S. Identification of SSRs and differentially expressed genes in two cultivars of celery (Apium graveolens $\mathrm{L}$.) by deep transcriptome sequencing. Hortic. Res. 1, 10 (2014).

65. Aubourg, S., Lecharny, A. \& Bohlmann, J. Genomic analysis of the terpenoid synthase (AtTPS) gene family of Arabidopsis thaliana. Mol. Genet. Genomics 267 730-745 (2002).

66. Falara, V. et al. The tomato terpene synthase gene family. Plant Physiol. 157, 770-789 (2011)

67. Yahyaa, M. et al. Identification and characterization of terpene synthases potentially involved in the formation of volatile terpenes in carrot (Daucus carota L.) roots. J. Agric. Food Chem. 63, 4870-4878 (2015).

68. Xia, E. H. et al. The tea tree genome provides insights into tea flavor and independent evolution of caffeine biosynthesis. Mol. Plant 10, 866-877 (2017).

69. Guan, R. et al. Draft genome of the living fossil Ginkgo biloba. GigaScience 5, 49 (2016).

70. El Baidouri, M. \& Panaud, O. Comparative genomic paleontology across plant kingdom reveals the dynamics of TE-driven genome evolution. Genome Biol. Evol. 5, 954-965 (2013).

71. Bohlmann, J., Meyer-Gauen, G. \& Croteau, R. Plant terpenoid synthases: molecular biology and phylogenetic analysis. Proc. Natl Acad. Sci. 95, 4126-4133 (1998).

72. Tholl, D. Terpene synthases and the regulation, diversity and biological roles of terpene metabolism. Curr. Opin. Plant Biol. 9, 297-304 (2006).

73. Yao, L. X. et al. Proteomic and metabolomic analyses provide insight into the off-flavour of fruits from citrus trees infected with 'Candidatus Liberibacter asiaticus'. Hortic. Res. 6, 31 (2019). 